\title{
Diversidad espacial y temporal de arañas en microhábitats de cultivos de Citrus sinensis (Rutaceae), Corrientes, Argentina
}

\author{
Helga Cecilia Achitte-Schmutzler ${ }^{1}$, Eduardo Adolfo Porcel $^{2}$ \& Gilberto Avalos ${ }^{1}$ \\ 1. Cátedra de Biología de los Artrópodos, Facultad de Ciencias Exactas y Naturales, Universidad Nacional del Nordeste, Avda. \\ Libertad 5470 (3400) Corrientes, Argentina; ceciliaachitte@hotmail.com, gilbertoa@exa.unne.edu.ar \\ 2. Departamento de Matemática. Facultad de Ciencias Exactas y Naturales y Agrimensura. Universidad Nacional del Nordeste. \\ Corrientes, Argentina; eporcel@exa.unne.edu.ar
}

Recibido 28-X-2017. Corregido 28-V-2018. Aceptado 13-IX-2018.

\begin{abstract}
Spatial and temporal diversity of spiders in microhabitats of Citrus sinensis orchards, (Rutaceae) Corrientes, Argentina. Arachnofauna studies from agroecosystems are crucial for implements spider as biological control agents. In this work we analyze the distribution of spider assemblages at three microhabitats (canopy, tree trunk and soil) in Citrus sinensis orchards (Rutaceae) in the Province of Corrientes, Argentina. Spiders were collected monthly using four sampling techniques: beating foliage, leaf litter sifting, pitfall traps and direct capture by hand. A total of 2160 samples were examined and 7194 spiders were collected (2462 on the canopy, 983 on trunk and 3749 on the floor) belonging to 34 families and 200 species/ morphospecies of Araneomorphae. There were significant differences among microhabitats and months in the structure of the spider communities, according to analysis of similarity (ANOSIM). The diversity was highest on the canopy, followed by soil and tree trunk. Araneidae, Salticidae and Anyphaenidae were the most abundant taxa in the canopy; the dominant species were Jessica erythostoma and Cheiracanthium inclusum, a predator of the leaf herbivore Phyllocnistis citrella (Lepidoptera) a pest of Citrus spp. The most abundant families on the tree trunk were Tetragnathidae, Hersiliidae and Theridiidae, Iviraiva pachyura was a species specialist in such microhabitat; and Leucauge venusta was an opportunistic species, because their abundance increaseds markedly in anthropic environments that modified by man. The most abundant families on the floor were Lycosidae, Linyphiidae and Coriniidae. In this habitat, Lycosa erythrognatha and Pardosa plumipedata were dominant species. The Chao-Jaccard similarity index was highest between the canopy and trunk $(\mathrm{J}-\mathrm{C}=0.95)$, likewise ordination by nonmetric multidimensional scaling (NMDS) revealed strong differentiation between assemblages of soil spiders and canopy and trunk spiders. Also, on the soil the temporary replacement of species was greater and the seriation method was significant $(\mathrm{z}=3.82, \mathrm{P}=0.00)$ therefore there was a succession of species during the year. The different species in each microhabitat probably avoided competition by using different hunting strategies and different ecological resources, in addition to specific patterns of temporal distribution. Our results suggest that anthropic environments, such as in Citrus orchards, tend to harbor populations of spiders able to adapt to those environments and interact in a balanced way in space and time.
\end{abstract}

Key words: microhabitat; Araneae; agroecosystems; oranges; biological control.

Achitte-Schmutzler, H. C., Porcel, E. A. \& Avalos, G. (2018). Diversidad espacial y temporal de arañas en microhábitats de cultivos de Citrus sinensis (Rutaceae), Corrientes, Argentina. Revista de Biología Tropical, 66(4), 1504-1518.

Argentina se destaca en la comercialización de cítricos a nivel mundial. En 2016 la producción de naranja alcanzó un millón 32 mil toneladas, de las cuales el $29 \%$ es producido en Corrientes (Danza, 2017). Indudablemente impera el uso de plaguicidas en dichos cultivos, entre los cuales predominan los insecticidas, algunos herbicidas y diversos tipos de fungicidas (Montti, Visciglio, Raviol, Subovich, \& Munitz, 2013). El uso de estos productos 
químicos constituye una problemática que impacta tanto a los sectores agrícolas como al ambiente y a la salud del ser humano debido a la presencia de residuos en los frutos y/o en sus productos industriales (Montti et al., 2013).

El control biológico de plagas es cada vez más aceptado como una alternativa contra el uso de insecticidas. Para su implementación se requiere de estudios preliminares de la biología y de la ecología sobre el complejo de enemigos naturales presentes en los agrosistemas (Bale, van Lenteren, \& Bigler, 2008). Entre estos organismos, las arañas adquieren cada vez mayor importancia debido a su capacidad de regular y evitar daños producidos por los diversos fitófagos que atacan a los cultivos (Barrientos, Villalba, Alvis-Davila, \& GarciaMar, 2010). De este modo, en los sistemas agrícolas los estudios referidos a Araneae han ido incrementándose progresivamente (Riechert \& Lockley, 1984; Nyffeler, Sterling, \& Dean, 1994; Lee \& Kim, 2001; Armendano \& González, 2011; Almada, Sosa, \& González, 2012). Particularmente en cultivos de cítricos se pueden citar los realizados por Carroll, 1980; Mansour \& Whitecomb, 1986; Van Den Berg, Dippenaar-Schoeman, Deacon, \& Anderson, 1992; Breene, Dean, \& Meagher, 1993; Benamú Pino, 2004; Avalos, Bar, Oscherov, \& González, 2013; que focalizan la fauna completa de arañas lo cual cobra importancia para el control de las poblaciones de algunos fitófagos (Foelix, 1996).

En los cultivos de cítricos las arañas son los depredadores más abundantes, por encima de otros grupos de depredadores considerados importantes como los neurópteros y los coccinélidos (Carroll, 1980; Amalin \& Peña, 1999). Diversas investigaciones verifican el importante papel que ocupan las arañas como controladores biológicos de plagas en dichos cultivos (Amalin, Peña, \& McSorley, 1996). Por ejemplo, Pardosa cribata (Lycosidae) tiene un potencial rol como controlador biológico sobre Ceratitis capitata (Diptera: Tephritidae) en España (Monzó, Mollá, Castañera, \&
Urbaneja, 2009). En Israel se han identificado las arañas predadoras de Phyllocnistis citrella (Lepidoptera), otra plaga importante de Citrus spp. (Argov \& Rossler, 1996). En el Sur de Florida varias especies de las llamadas "arañas de saco" (Eutichuridae) contribuyen al control de las afecciones causadas por lepidópteros, ácaros y trips (Amalin, Peña, McSorley, Browning, \& Crane, 2001).

Los patrones de distribución de las arañas varían entre tres microhábitats básicos: el follaje, el tronco y el suelo (Szinetar \& Horvath, 2005; Ziesche \& Roth, 2008; Michel \& Winter, 2009). No obstante, varios trabajos que analizan el ensamble de arañas en Citrus spp abarcan un microhábitat en particular, por ejemplo el suelo o el follaje (Amalin \& Peña, 1999; Elizondo Solís, 2002; Barrientos et al., 2010; Kacar, 2015).

Por otra parte, la estacionalidad es un factor que incide en la riqueza y abundancia de las comunidades de arañas (Wolda, 1988; Rodrigues, Mendonca Jr., Rodrigues, \& Ott, 2015) sin embargo pocas investigaciones (Carroll, 1980; Mansour, Ross, Edwards, Whitcomb, \& Richman, 1982) lo consideran al analizar los ensambles de arañas en cultivos de especies perennes. Por ejemplo, los estudios realizados en cultivos de manzanos (Patrick \& Canard, 1997), de almendros (Orellana, Ávila, \& Estrada, 2012) y de olivos (Marín Loayza, Herrera, \& Páez, 2013) los cuales abarcan solo los meses estivales.

Un primer estudio de la araneofauna en cultivos de $C$. sinensis en Corrientes, Argentina, analizó si los patrones de diversidad, riqueza, abundancia y estructura de los gremios de arañas varían en función del tratamiento de las parcelas (parcelas con riego y sin riego) (Avalos et al., 2013). En el presente trabajo se analizaron los patrones de distribución espacial y temporal de las comunidades de arañas en los microhábitats follaje, tronco y suelo, en los cultivos de C. Sinensis; se comparó la abundancia y riqueza específica, así como las fluctuaciones temporales en cada uno de los mismos. 


\section{MATERIALES Y MÉTODOS}

La investigación se llevó cabo en la Estación experimental del INTA Bella Vista, Provincia de Corrientes, Argentina, (28 $26^{\prime} \mathrm{S}$ - 58 55' W). El Departamento Bella Vista se halla a $70 \mathrm{~m}$ sobre el nivel del mar, el suelo es franco-arenoso, y el régimen pluviométrico es, en promedio, de $1300 \mathrm{~mm}$ anuales (Avalos et al., 2013). La temperatura máxima absoluta es de $33{ }^{\circ} \mathrm{C}$, la mínima absoluta de $8.5^{\circ} \mathrm{C}$, y la media es $20.5^{\circ} \mathrm{C}$ (Avalos et al., 2013). El área de estudio pertenece al Distrito Oriental Húmedo de la Provincia Fitogeográfica Chaqueña (Cabrera \& Willink, 1973), Subregión Chaqueña, Provincia Chaco (Morrone, 2001).

Se estudió una parcela de cultivos de naranjos Citrus sinensis (L) Osbeck, con 784 plantas. La edad aproximada de los ejemplares es 17 años y comprenden árboles vigorosos, con una altura media de $3 \mathrm{~m}$. Son cultivos convencionales con tratamientos de riego, fertilizantes e insecticidas.

El sitio de muestreo se encuentra rodeado por áreas modificadas por el hombre: bordes con plantaciones de eucaliptos alternados con pastizales y viviendas. Dentro del cultivo la vegetación es muy escasa, puesto que las malezas interfieren con las labores de cosecha, poda, pulverizaciones, fertilizaciones y son huéspedes de plagas y enfermedades que luego pasan al cultivo (trips, pulgones, Tristeza, Satsuma dwarf, CVC) (Gómez, 2016), estas son controladas con herbicidas y desmalezadoras.

Cabe aclarar que el tratamiento de malezas fue aplicado unos días antes de comenzar esta investigación, luego fue suspendido hasta finalizar el muestreo.

Actividades de campo: Los muestreos se realizaron mensualmente durante un año, (desde octubre de 2005 hasta septiembre de 2006). Se seleccionaron veinte plantas de naranjo en base a un muestreo sistemático. En cada árbol se seleccionaron al azar tres sectores de cada microhábitat (follaje, tronco y suelo) en los cuales se recolectaron las arañas mediante las siguientes técnicas:
Para el follaje: (a) Agitación de follaje: consistió en 15 sacudidas vigorosas de un sector de la copa de la planta, seleccionado al azar, sobre un paraguas entomológico de 70 $\mathrm{cm}$ de largo por $60 \mathrm{~cm}$ de ancho. (b) Captura manual nocturna: se capturaron directamente con pinzas las arañas visualizadas en el follaje durante 5 minutos en cada sector seleccionado de la copa.

Para el tronco: (a) Captura manual diurna y nocturna: para poder visualizar la arañas del tronco, aplicamos una técnica que llamamos "acariciar el tronco", que consiste en pasar la mano sobre el mismo desde abajo hacia arriba y observar la presencia de arañas cuando estas se movían, las cuales se capturaron durante 5 min en cada horario y planta.

Para el suelo: (a) Pitfall: se instalaron tres trampas de caída en la parte basal de cada árbol, formando un triángulo alrededor de él, a una distancia de $1.50 \mathrm{~m}$. Las trampas consistieron en recipientes plásticos de $900 \mathrm{ml}$ con una abertura de $11 \mathrm{~cm}$ de diámetro, con etilenglicol diluido en agua en proporción 1:10 y gotas de detergente. Las mismas se enterraron a nivel del suelo sin cubierta y permanecieron activas durante 48h. (b) Tamizado de la hojarasca: se tamizaron superficies de $0.50 \mathrm{~m}^{2}$ de hojarasca sobre un lienzo blanco de $3 \mathrm{~m}^{2}$ con un tamiz de $10 \times 15 \mathrm{~mm}$ de apertura de malla, en tres lugares cercanos a cada árbol seleccionado.

De este modo, en cada microhábitat se obtuvieron tres muestras que incluyen una recolección diurna y una nocturna de arañas. Así, la unidad de muestreo queda representada por un conjunto complementario de métodos diferentes cada uno empleado durante un tiempo concreto (Jiménez-Valverde \& Hortal, 2003). En total se obtuvieron 2160 muestras (3 muestras x 3 microhábitats x 20 árboles X 12 meses).

Actividades de laboratorio: Los ejemplares fueron conservados en alcohol $70 \%$. Para determinar las familias de Araneae se utilizó la clave de Ramírez (1999) y para las especies se consultaron claves y trabajos de revisiones disponibles en el World Spider Catalog (2018). 
Los individuos adultos que no han podido determinarse a nivel específico fueron catalogados como (morfo) especies (Krell, 2004) de acuerdo a las diferencias observadas en las genitalias.

Análisis de datos: En cada microhábitat y durante los meses de muestreo se calculó la riqueza y la abundancia de especies y familias de Araneomorphae. Además, para cada microhábitat se calcularon los índices de dominancia de Simpson y de equitatibilidad de Pielou (Moreno, 2001).

La eficiencia del muestreo en cada microhábitat fue analizada mediante las curvas de acumulación de especies observadas y estimadas (rarefacción), con el estimador Mao Tau (Colwell, Mao, \& Chang, 2004) con sus respectivos intervalos de confianza (95\%). Como medida del esfuerzo de muestreo se empleó el número de muestras agrupadas (para cada árbol se agruparon las muestras obtenidas del follaje, del tronco y del suelo). Los datos se ajustaron con el modelo de Clench y el número esperado de especies se calculó como el cociente $\mathrm{a} / \mathrm{b}$ de los parámetros del modelo. Luego se comparó dicho número con el de las especies realmente observadas. Para este análisis se empleó el número de muestras como medida del esfuerzo de muestreo.

La distribución de la abundancia se ajustó a los modelos paramétricos de serie geométrica, serie logarítmica y la distribución lognormal, mediante la prueba de bondad de ajuste $X^{2}$ (Sokal \& Rohlf, 1995).

Para detectar diferencias en la estructura de la comunidad entre los distintos microhábitats y por estaciones se utilizó el análisis de similitud (ANOSIM) con 9999 permutaciones (Clarke, 1993). Además, se realizó un análisis de porcentaje de similitud (SIMPER) a fin de determinar qué especies de arañas contribuyen más a la disimilitud espacio-temporal. Para este último análisis se consideraron aquellas especies que explican al menos un $50 \%$ de las diferencias entre grupos.

La diversidad beta espacial entre los tres microhábitats y la beta temporal entre los meses de muestreo se analizó mediante el índice Chao-Jaccard, el cual tiene en cuenta las especies compartidas no vistas y es más apropiado para la evaluación de la similitud entre muestras de diferentes tamaños con numerosas especies raras (Chao, Chazdon, Colwell, \& Shen, 2005). Estos análisis se realizaron con el programa EstimateS v.9.1.0 (Colwell, 2013).

Se realizó un análisis de escalamiento multidimensional no métrico (NMDS) basado en la medida de distancia de Bray-Curtis, a fin de observar patrones de agrupación de las especies y sus abundancias en los microhábitats. Para este análisis se seleccionaron todas las especies y sus abundancias se jerarquizaron del siguiente modo: alta (con más de 200 individuos), media (entre 100 y 200 individuos) y baja (con menos de 100 individuos).

La variación de especies a escala temporal dentro de cada microhábitat se analizó utilizando el método de seriación restringida (Brower \& Kile, 1988), el cual efectúa una simulación Monte Carlo creando 30 matrices aleatorias y prueba si el gradiente temporal observado en los datos originales es diferente de uno aleatorio.

Los análisis se realizaron con el software PAST (Hammer, Harper, \& Ryan, 2001) y para las pruebas de hipótesis se utilizó un nivel de significación $\alpha=0.05$.

\section{RESULTADOS}

Se recolectaron 7194 individuos pertenecientes a 35 familias y 200 (morfo) especies de Araneomorphae. La mayor riqueza se halló en el follaje, seguida por el suelo y el tronco (Apéndice 1).

Se obtuvieron valores muy cercanos de dominancia en los tres microhábitats (Apéndice 1). En follaje Jessica erythostoma fue la especie dominante, en tronco Ivyraiva pachyura y en suelo Lycosa erythrognatha.

La comparación de los resultados con el número de especies estimado por el modelo de Clench refleja que el muestreo total de arañas en todo el cultivo ha sido exitoso. En el suelo se registraron el $96.9 \%$, mientras que en el follaje 
y en el tronco se hallaron el $86.7 \%$ y el 69.5 $\%$ de las especies estimadas (Apéndice 1). Esta situación se observa en las curvas de acumulación donde el follaje y el tronco no alcanzan la asíntota (Fig. 1). Asimismo la distribución de la abundancia en los tres microhábitats mostró un mejor ajuste al modelo log-normal (Apéndice 1).

Cada microhábitat presentó diferentes ensambles de arañas, así en el follaje predominaron Araneidae, Anyphaenidae y Salticidae, en el tronco Tetragnathidae, Hersiliisdae y Theridiidae; y en el suelo Lycosidae, Linyphiidae y Corinnidae.

Algunas familias fueron encontradas en un microhábitat particular, por ejemplo en el tronco se hallaron Selenopidae $(\mathrm{N}=1)$ y Uloboridae $(\mathrm{N}=2)$ y en el suelo Caponiidae $(\mathrm{N}=3)$, Miturgidae $(\mathrm{N}=41)$, Oonopidae $(\mathrm{N}=26)$, Palpimanidae $(\mathrm{N}=2)$, Prodidomidae $(\mathrm{N}=155)$, Titanoecidae $(\mathrm{N}=11)$ y Zodariidae $(\mathrm{N}=120)$.
También a nivel específico muchas especies fueron abundantes en un único microhábitat mientras que en los otros fueron escasas o estuvieron ausentes (Apéndice 2). Además, en cada microhábitat se encontraron especies únicas, por ejemplo en follaje se obtuvieron 15 especies de Areneidae, tres de Oxyopidae, dos de Salticidae y dos de Theridiidae que no se hallaron en el tronco ni en suelo. De igual forma, ocho especies de Araneidae y una de Uloboridae solo se hallaron en el tronco y 31 especies de diferentes familias en el suelo (entre ellas Corinnidae, Gnaphosidae, Linyphiidae, Lycosidae, Philodromidae, Salticidae, Theridiidae, y las familias nombradas anteriormente halladas únicamente en este microhábitat).

No obstante, algunas especies fueron abundantes en dos microhábitats, por ejemplo Metaltella simoni (Amphinectidae) de la que se capturaron 55 individuos en follaje y 68 en suelo; Dictynidae especie morfo 2 con 32

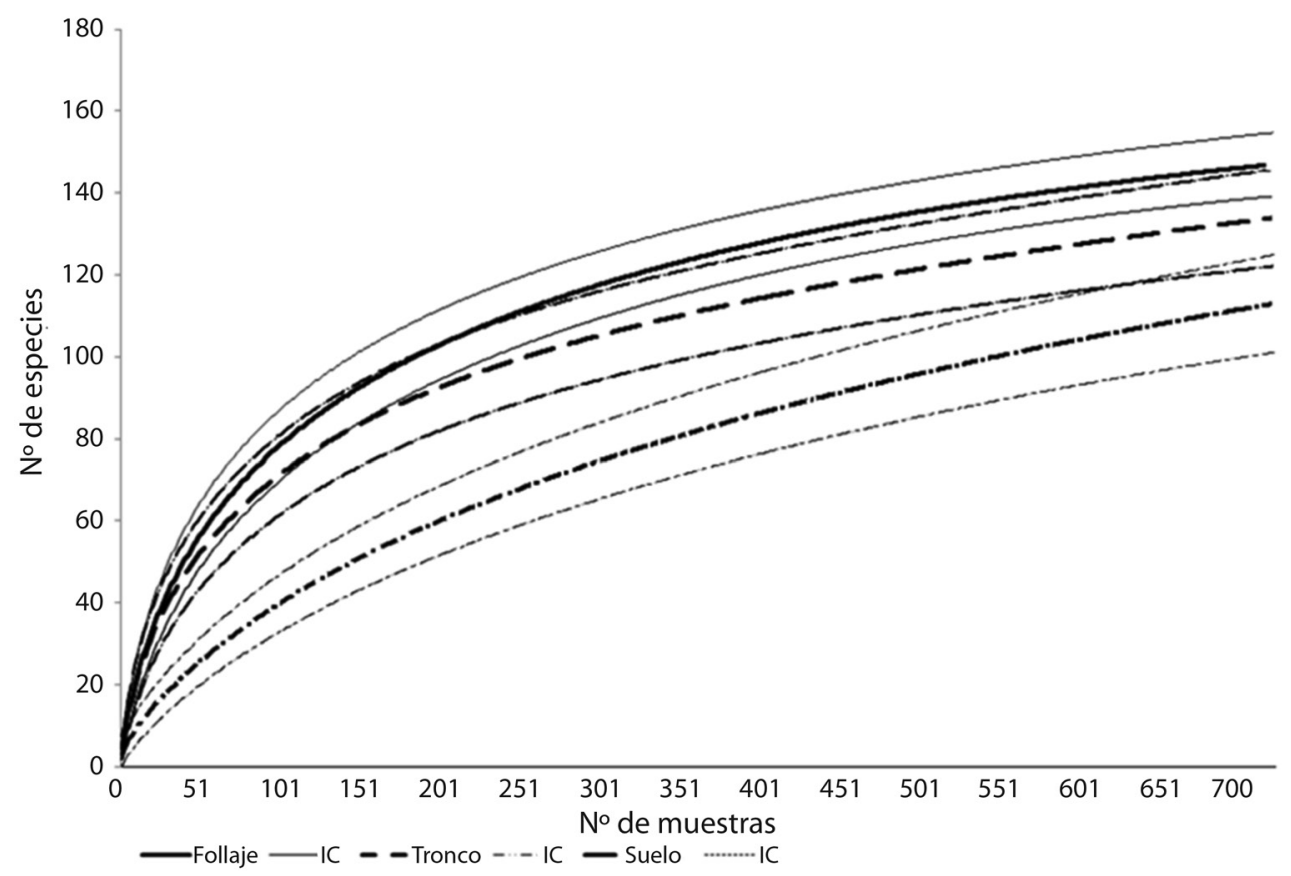

Fig. 1. Curvas de acumulación (Mao Tau) y sus intervalos de confianza, de la riqueza de arañas observada en los tres microhábitats (Follaje, Tronco y Suelo) de Citrus sinensis.

Fig. 1. Accumulation curves (Mao Tau) and confidence intervals, of observed spiders richness at three microhabitats (canopy, trunk and soil) of Citrus sinensis. 
individuos en el follaje y 17 en el suelo; Frigga quintensis y otras dos especies morfo de Saltícidos también se encuentraron en estos dos microhábitats con más de 10 individuos. Asimismo en follaje-tronco se hallaron Senoculus sp. (Senoculidae), Polybetes rapidus (Sparassidae) y Leucauge venusta (Tetragnathidae).

En los tres ambientes en forma simultánea se hallaron Cheiracantium inclusum y Cryptachaea altiventer; sumadas a muchas especies con muy baja abundancia (con menos de diez individuos en cada microhábitat) de las siguientes familias: Araneidae $(\mathrm{S}=2)$, Mimetidae $(\mathrm{S}=1)$, Oxyopidae $(\mathrm{S}=1)$, Philodromidae $(\mathrm{S}=1)$, Salticidae $(\mathrm{S}=5)$ y Theridiidae $(\mathrm{S}=5)$.

Diversidad en cada microhábitat: El análisis de similitud (ANOSIM) detectó diferencias estadísticamente significativas en la estructura de la comunidad entre los tres microhábitats (Factor AMBIENTE $\mathrm{R}=0.2826 \mathrm{P}=$ 0.0001 ). De acuerdo al análisis SIMPER varias especies contribuyeron a estas diferencias entre los microhábitats; por ejemplo L. venusta e $I$. pachyura son las especies que más contribuyen a la diferenciación del tronco, Araneus uniforme y L. erythrognatha a la diferenciación del follaje $\mathrm{y}$ del suelo respectivamente (Apéndice 3).

El análisis de Chao-Jaccard mostró mayor similitud entre el follaje y el tronco ( $\mathrm{J}-\mathrm{C}=$ 0.95), mientras que entre el follaje-suelo y el tronco-suelo se obtuvieron valores menores ( 0.49 y 0.50 respectivamente). Por tanto entre estos últimos microhábitats hay mayor recambio de especies. El análisis de NMDS confirmó los resultados anteriores (con un stress de 0.11), ya que mostró dos grandes grupos, uno con las especies predominantes del suelo, y el otro grupo con las especies del tronco y del follaje. Además, el eje 2 separa a las especies según su abundancia, en forma creciente de arriba hacia abajo en el gráfico (Fig. 2).

Diversidad temporal en los microhábitats: Según el análisis de similitud (ANOSIM)

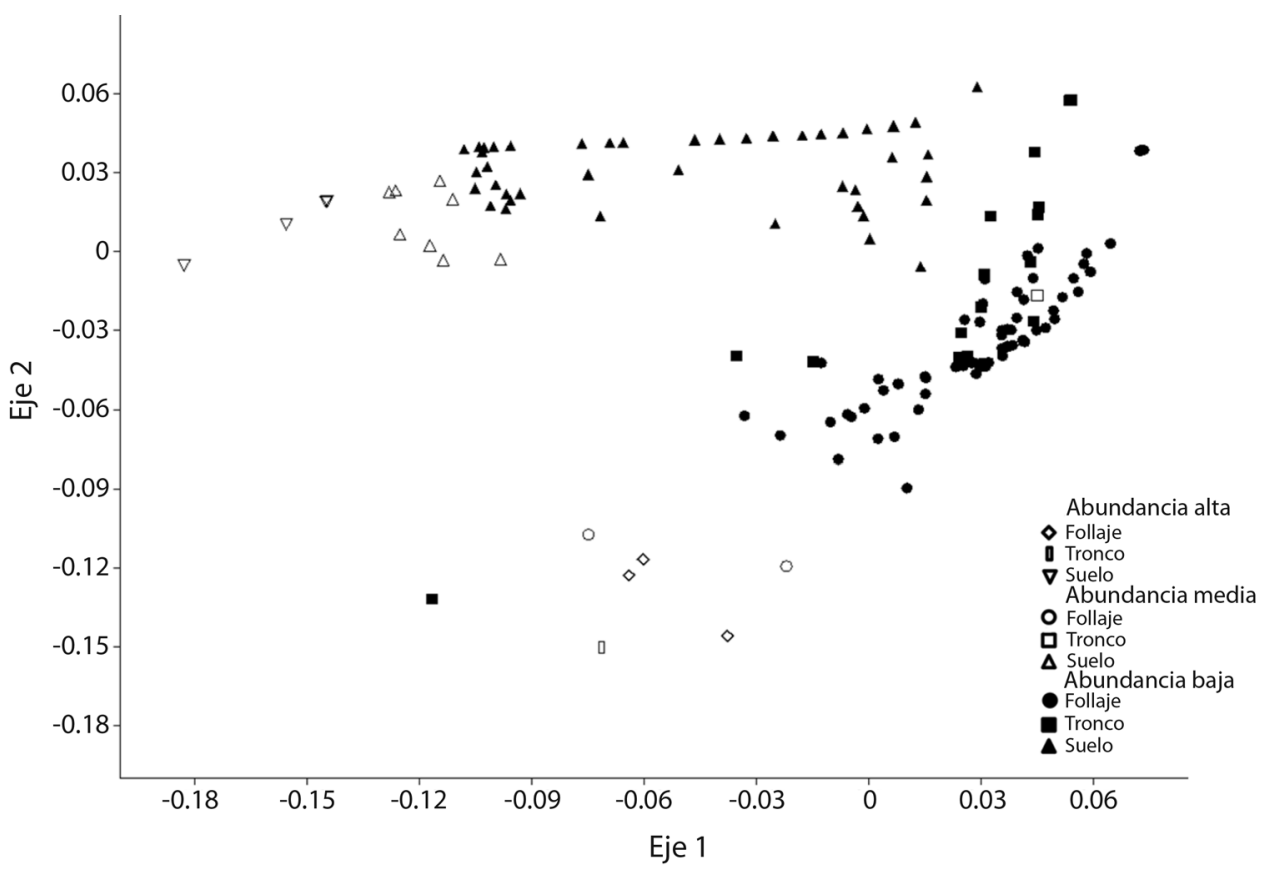

Fig. 2. Diagrama de ordenación de multivariados de ordenamiento Escalado Multidimencional no Métrico (NMDS) basado en el índice de similitud de Bray-Curtis, para la abundancia de arañas en los microhábitats de los cultivos de Citrus sinensis. Fig. 2. Ordination plot of non-metric multidimensional scaling (NMDS) based on the Bray-Curtis similarity index, for the abundance of spiders in the microhabitats of Citrus sinensis orchards. 
hubo diferencias significativas en la estructura de la comunidad de arañas entre los meses del año en que se realizó el muestreo (Factor MES $\mathrm{R}=0.13568 \mathrm{P}=0.0001)$. Las especies que más contribuyen en la diferenciación entre las estaciones del año pertenecen a las familias Lycosidae y Linyphiidae (Apéndice 3).

El recambio de especies fue mayor en el suelo, seguido por el follaje y el tronco. Los meses con mayor recambio fueron abril y julio para el suelo, julio y agosto para el follaje $(\mathrm{J}-\mathrm{C}=0.53)$, y marzo y abril $(\mathrm{J}-\mathrm{C}=0.64)$ para el tronco.

En el follaje los meses con mayor abundancia fueron los de invierno, primavera y principio de verano con predominancia de A. uniformis, C. altiventer y J. erythrosoma respectivamente (Apéndice 4); y la mayor cantidad de especies se registró en otoño y primavera. En el tronco la mayor abundancia se registró en primavera y principio de verano con predominancia de L. venusta, coincidiendo también con la mayor riqueza de especies. En el suelo, tanto la abundancia como la riqueza de especies fueron mayores a fines de primavera (con predominancia de Dubiaranea sp., Linyphiidae) y principio de verano (con predominancia de L. erythrognatha) (Apéndice 4). En esta figura, además, se observa un decaimiento de las abundancias de las especies dominantes del suelo en el mes de octubre, en coincidencia con el tratamiento aplicado contra las malezas en dicho mes. En el mes siguiente (noviembre) se observa un aumento abrupto de la abundancia de Dubiaranea sp., la cual llega a ser la especie dominante hasta que vuelve a normalizarse la abundancia de L. erythrognatha, Pardosa plumipedata y Lobizón humilis.

El patrón de presencias y ausencias de especies a lo largo de los 12 meses de estudio, analizado mediante el método de seriación solo resultó significativo en el suelo $(\mathrm{z}=3.82, \mathrm{P}=$ $0.00)$ no así en el follaje $(\mathrm{z}=0.75, \mathrm{P}=0.44) \mathrm{ni}$ en el tronco $(\mathrm{z}=-0.82, \mathrm{P}=0.41)$. Dicho patrón implica que algunas especies están durante los meses cálidos y luego se ausentan, por ejemplo especies de varias familias como Anyphaenidae, Araneidae, Caponiidae, Corinnidae,
Ctenidae, etc.; otras están durante todo el año (mayormente especies de Lycosidae) y otras solamente en los meses fríos, entre ellas varias especies de Anyphaenidae, Trachelidae, Dictynidae, etc. (Apéndice 5).

\section{DISCUSIÓN}

La mayoría de las familias halladas en esta investigación se encuentran entre las más numerosas en los agrosistemas (Nyffeler et al., 1994), a excepción de Corinnidae y Anyphaenidae que también tuvieron una importante representación numérica en este trabajo. Particularmente sobresale la abundancia de Anyphaenidae, ya que arañas de esta familia no se encontraron en los Citrus spp. de otros países como en España (Barrientos et al., 2010), en Sudáfrica (Van Den Berg et al., 1992) y en India (Keswani, 2014)

Los resultados obtenidos reflejan diferencias en la composición específica de arañas en cada microhábitat de Citrus sinensis, con especies dominantes para el follaje, el tronco y el suelo. El ajuste a la distribución log normal sugiere que estos ensambles de arañas son estables, con bajo estrés y sometidas a la influencia de escasos factores (Moreno, 2001). Este tipo de distribución de la fauna de arañas también se verifica en ambientes naturales y boscosos (Rubio, Corronca, \& Damborsky, 2008; Avalos, Damborsky, Bar, Oscherov, \& Porcel, 2009; Almada, 2014; Achitte-Schmutzler, Avalos, \& Oscherov, 2016). Dado que la mayor heterogeneidad espacial en estos ambientes proporciona gran variedad de microhábitats $\mathrm{y}$, por tanto, hay mayor cantidad de especies (Begon, Harper, \& Townsend, 1995), los Citrus estudiados podrían ser considerados como un ambiente heterogéneo con microhábitats bien definidos (follaje, tronco y suelo) cada uno de los cuales con una elevada riqueza específica.

En el follaje predominaron Araneidae, Theridiidae y Salticidae, estas familias son comunes en ambientes naturales como bosques y pastizales y en ambientes antropizados como las forestaciones (Avalos, Achitte-Schmutzler, \& De los Santos, 2018); también se encuentran 
entre las más abundantes en cultivos de Citrus de otros países (Barrientos et al., 2010). Se trata en realidad de familias con elevado número de especies que se han adaptado a diversos ambientes (World spider Catalog, 2018). Cardoso, Pekár, Jocqué, y Coddington (2011) sostienen que, si bien la composición específica varía, en hábitats con estructuras similares se espera que la composición de gremios sea similar. Sin embargo, en los cultivos de Citrus en India predominó Thomisidae cuya estrategia de caza difiere al de estas familias mencionadas (Keswani, 2014).

Por otra parte, mientras que en el follaje de los cultivos de C. sinensis de España Linyphiidae ocupa el segundo puesto en cuanto a abundancia, superando a Araneidae (Barrientos et al., 2010), en este estudio fueron muy escasos en este microhábitat pero abundantes en el suelo.

En este trabajo el follaje presenta un ensamble de arañas estable, con especies que predominan durante todo el año y otras con menor abundancia que aparecen solo algunos meses. Estas especies pertenecen a distintos gremios, entre ellos se encuentran las constructoras de telas orbiculares (Araneidae) y de telas irregulares (Theridiidae), las vagabundas de la vegetación (Anyphaenidae) y las cazadoras al acecho (Salticidae). Estos grupos son los más representativos del ensamble, fundamentalmente aquellas especies que se encontraron durante todos los meses, lo cual evidencia que este microhábitat es óptimo para el desarrollo completo del ciclo de vida de estas arañas. En concordancia con Henschell y Lubin (1997) quienes sostienen que la complejidad estructural de las ramas provee a las arañas mayores sitios de refugio y un óptimo microclima, y con Elizondo-Solís (2002) quien sostiene que estas condiciones favorables para las arañas aumentan proporcionalmente con la antigüedad de los cultivos; de igual manera, creemos que los Citrus estudiados han ido estableciendo durante casi dos décadas un sistema integrado de refugios, sitios de forrajeo, de reproducción, etc. y de artrópodos que hacen uso de estos sitios. Coincidimos con Elizondo-Solís (2002) quien confirma que la diversidad de insectos y arañas en $C$. sinensis interactúan de manera equilibrada en este sistema.

Entre las especies representativas del follaje se encuentran aquellas de posible interés para el control biológico de plagas; por ejemplo según Avalos, Oscherov, y González (2015) J. erythrostoma (Anyphaenidae) podría tener un rol semejante a Hibana velox (Anyphaenidae) como potencial predador de Phyllocnistis citrella (lepidóptera), plaga que ataca las hojas de Citrus spp. (Amalin \& Peña, 1999; Amalin et al., 2001). En la misma situación se encontraría el género Aysha, aunque hallada en menor número en este estudio, este género también tiene una especie (A. gracilis) que se alimenta de huevos de Heliothis virescens (Noctuidae), cuya larva es plaga de varios cultivos (McDaniel \& Sterling 1982).

También fue representativa del follaje $A$. uniformis, que tiene importancia debido a su preferencia alimentaria por los hemípteros (Avalos et al., 2015), una de las plagas de mayor impacto en Cítricos (Elizondo-Solís, 2002). Y C. inclusum, otra predadora de las larvas de P. citrella (Amalin et al., 2001). Asimismo, en el follaje de $C$. sinensis de España fue abundante $C$. mildei (Barrientos et al., 2010) que probablemente también ejerza algún control sobre esta plaga en dicho país, donde $P$. citrella, detectada por primera vez en 1993, colonizó todas las zonas citrícolas del país en un año (Garijo \& García, 1994). Por su parte, en el follaje de los Citrus de India fue hallada otra especie de este género, C. inornatum, que además fue capturada en la hojarasca del cultivo (Keswani, 2004).

En coincidencia con Szinetár \& Horváth (2005) quienes sostienen que el microhábitat del tronco alberga especies adaptadas morfológica, fenológica y fisiológicamente a vivir sobre o bajo la corteza, en esta investigación se registró a I. pachyura en todos los meses de muestreo. Las características morfológicas de esta especie (cuerpo aplanado, patas largas y coloración semejante al tronco) en combinación con la estrategia de caza que utiliza (por emboscada) favorecen el habitáculo de esta 
araña en este microhábitat donde pueden pasar desapercibidas. Por otra parte, L. venusta que presenta un opistosoma globoso y brillante y construye telas para cazar, tuvo una representación importante en el tronco, pero también fue hallada, aunque en menor abundancia, en el follaje. Es interesante destacar que, según diversas investigaciones (Avalos et al., 2009; Escobar, Avalos, \& Damborsky, 2012; AchitteSchmutzler et al., 2016) esta especie disminuye su abundancia de manera notable en ambientes naturales más conservados, sobre todo en bosques. Por ello, consideramos que se trata de una especie oportunista, debido a que su abundancia se incrementa notablemente en sitios modificados por el hombre; estos ambientes alterados donde muchas especies pierden sus hábitats, parecen ser propicios para la colonización del género Leucauge (Avalos et al., 2018).

Respecto a las familias halladas únicamente en el tronco, como Selenopidae y Uloboridae, estas tuvieron una abundancia muy baja, mientras que en los cultivos de $C$. sinensis de Uruguay estuvieron ausentes (Benamú Pino, 2004).

Particularmente Uloboridae también fue poco abundante en otros cultivos (Dean, Sterling \& Horner, 1982) y no se registró en alfalfa y algodón (Armendano \& González, 2011; Almada et al., 2012). No obstante abundan en el follaje de los cultivos de Citrus en la India (Keswani, 2014) y en otros ambientes como en las selvas tropicales (Florez, 2000), en los bosques del Distrito Oriental Húmedo del Chaco (Avalos et al., 2009; Escobar et al., 2012) y en plantaciones de pinos (Avalos et al., 2018). Consideramos que, al igual que otras especies con baja proporción de individuos, podrían tratarse de especies turistas propias de otros microhábitats, que se encuentran temporal y efímeramente en este cultivo estudiado. En muchos inventarios de arañas las especies raras son catalogadas como singletons o dobletons (Toti, Coyle, \& Miller, 2000; Sorensen, Coddington, \& Scharff, 2002) cuando en realidad en algunos casos podrían tratarse de especies cripticas o con distribuciones irregulares o bien ser consecuencia de una insuficiencia del protocolo de muestreo empleado (Rubio et al., 2008).

Por otra parte, el suelo presenta una composición específica bastante diferente si comparamos con el follaje y el tronco, de acuerdo al patrón de agrupamiento observado en el NMDS y la elevada disimilitud respecto a ambos microhábitats mencionados. $\mathrm{Y}$ es que varias familias se hallaron solamente en este microhábitat, la mayoría de ellas son errantes (Lycosidae, Palpimanidae Prodidomidae Zodariidae, Caponiidae) y otras tejen telas sábana (Linyphiidae, Titanoecidae).

Entre las arañas errantes se destacaron las Lycosidae que son muy comunes en áreas abiertas antropizadas (Costa, Pérez-Miles, Gudynas, Prandi, \& Capocasale, 1991) como así también en ambientes de sabanas, bosques exóticos y pajonales (Rubio, Minoli, \& Piacentini, 2007; Almada \& Sarquis, 2016). Esta familia presenta arañas deambuladoras muy activas sobre la superficie terrestre (Pearce \& Zalucki, 2006) y especies que no están restringidas a un tipo de hábitat particular (Martin \& Major, 2001); por lo que probablemente también abunden en los alrededores del cultivo.

Esta familia también fue abundante en los cultivos de Citrus de España con predominio de Paradosa Cribata, un eficiente predador de una de las plagas más devastadoras, Ceratitis capitata (Diptera: Tephritidae) (Monzó et al., 2009). En esta investigación fue abundante $P$. plumipedata, por lo cual podría ser importante como regulador de esta plaga en estos Citrus.

Así mismo, se registró una abundancia considerable de L. humilis, esta especie mostró una distribución restringida en ambientes de sabana en Corrientes (Rubio et al., 2007) y según estos autores podría ser utilizada como especie indicadora biológica en estos ambientes al compararlo con un bosque higrófilo. No obstante, los cultivos de Citrus también parecen propicios para esta especie en la que se encontraron durante todos los meses de muestreo, aunque no se realizaron estudios en los ambientes aledaños al cultivo.

Por su parte, Linyphiidae, la segunda familia más abundante en este estudio, es 
comúnmente encontrada en los sistemas con disturbio (Schmidt \& Tscharntke, 2005). Ruzicka (1987) afirma que Lycosidae y Linyphiidae no superan juntos el $45 \%$ de las capturas en áreas protegidas, mientras que alcanzan el 85 $\%$ en áreas distorsionadas por el hombre. En este trabajo ambas familias alcanzaron el $54 \%$ de las capturas, lo que sugiere que hay cierto grado de perturbación antrópica en relación con los valores obtenidos (54.41\%) por PérezMiles, Simó, Toscano-Gadea y Useta (1999) en ambientes urbanizados. En parte, este resultado podría ser consecuencia del control de malezas que se efectúa en estos cultivos, lo cual impide el crecimiento de la vegetación baja y esto facilita el desplazamiento de las arañas errantes (Díaz Porres, Rionda; Duhour, \& Momo, 2014).

Desde el punto de vista temporal, el recambio de especies tanto en el follaje como en el suelo y el tronco, dan cuenta de la estabilidad que presentan estas comunidades de arañas en estos cultivos, donde algunas especies aparecen en estaciones cálidas, otras en estaciones frías y otras se han adaptado de tal manera que permanecen durante todos los meses, con individuos en diferentes estadios, lo que sugiere que pueden tener varias generaciones al año manteniendo una población casi constante, en concordancia con Jiménez y Tejas (1996).

$\mathrm{El}$ mayor recambio observado en invierno en el follaje puede corresponderse al período de fenología de estos cultivos que comenzó a mediados de agosto (Molina et al., 2005), además según estos autores, no hubo registro de heladas que puedan dañar la producción. La diversidad de insectos polinizadores atraídos por las flores, ligeramente aromáticas, de $C$. sinensis provee nuevas y abundantes presas disponibles para las arañas.

En el suelo el análisis temporal mostró especies que en general no se esperaría encontrar en dicho microhábitat, como por ejemplo algunas especies de Anyphaenidae que están ausentes en suelos boscosos (Grismado et al., 2011). Esto remarca la importancia de la estacionalidad en estudios ecológicos, ya que las arañas ocupan una gran cantidad de nichos espaciales y temporales (Quijano \& Neis Martínez, 2015). Además, las variaciones estacionales pueden determinar la composición y establecer diferencias entre los miembros de los gremios de arañas (Nentwig, 1993).

La distribución de las especies durante los meses muestreados en follaje y tronco muestran picos de abundancia en diferentes meses para las distintas especies. En cambio en el suelo, L. erytrognatha es la que domina en todos los meses exceptuando el mes de octubre, durante el cual se aplicó el tratamiento contra las malezas. Consideramos que las alteraciones químicas y mecánicas debido a dicho tratamiento tuvieron consecuencias notorias en la estructura de la fauna de arañas del suelo en este mes. La disminución drástica de especies dominantes podría suponer la mortalidad de los individuos de manera directa, o bien por modificación del microhábitat y la destrucción de nidos y ootecas (Riechert \& Lockley, 1984). Puesto que las arañas migran de ambientes desfavorables (condiciones de microclima, alimentos, etc.) hacia ambientes con condiciones favorables (Heublein, 1983), consideramos que algunas especies pudieron huir hacia los ambientes próximos como los pastizales y eucaliptos, y luego recolonizar el cultivo, sobre todo en el caso de las Lycosidae que se desplazan activamente, recorren grandes distancias y pueden ocupar ambientes efímeros o estacionales (Uetz, 1976). Además, estudios de marcado y recaptura han demostrado que algunos licósidos presentan diferentes respuestas de comportamiento dependiendo del tipo y calidad de hábitat y son capaces de desplazarse más de $5 \mathrm{~m}$ por día (Rubio et al., 2007). Estas arañas en poco tiempo recuperaron su abundancia y volvieron a ser dominantes en este microhábitat. No obstante, la baja abundancia de Lycosidae favoreció el aumento de la población de Dubiaranea sp.

Los ensambles de arañas en cada microhábitat podrían estar limitados por la competencia y la depredación de acuerdo a Jackson, PeresNeto y Olden (2001). Sin embargo, las familias de arañas en cada microhábitat probablemente eviten la competición al utilizar distintas 
estrategias de caza y distintos recursos ecológicos (Cardoso et al., 2011). Por ejemplo, según Nentwig (1987) las orbiculares presentan una amplia gama de alimentación en la que incluyen insectos con cutícula muy esclerotizada, con protección química y de comportamiento agresivo, pero son bastantes ineficientes para cazar polillas y mariposas (Eisner, Alsop, \& Ettershank, 1964). Los lepidópteros también escapan de las frágiles telas sábanas de Lyniphiidae (Turnbull, 1960) y constituyen gran parte de su dieta numerosos insectos pequeños como dípteros, homópteros, himenópteros y heterópteros (Turnbull, 1960; Nyffeler \& Benz, 1981). De igual manera, A. uniformis y arañas de la familia Anyphaenidae que predominaron en esta investigación, podrían utilizar estos recursos.

A su vez, la competencia entre los miembros de una familia podría estar condicionada por otros factores como el período del día (diurno-nocturno) en que se efectúa la caza. Aun así, dentro de un período del día, otros factores intervienen para evitar la competencia; por ejemplo, las Araneidae del follaje que estuvieron representadas por diversas especies de Araneus, Alpaida, Acasecia, Aculepeira, Cyclosa, Eustala (entre otras), utilizan la misma estrategia de caza, son nocturnas y pueden encontrarse en sus telas las mismas presas (Nyffeler \& Benz, 1989); sin embargo, estas arañas podrían evitar la competencia intraespecífica mediante la selección de diversos tipos o número de presas; como consecuencia de las diferencias morfológicas entre los géneros ya que existe una relación entre el tamaño del depredador y el de la presa seleccionada (Nyffeler \& Benz, 1981; Wise, 1993); y la estructura de la tela, lo cual implica tanto su tamaño como el espacio dentro de la malla (Nyffeler \& Benz, 1989).

Asimismo, las Linyphiidae halladas en gran abundancia en el suelo, reducen la probabilidad de las interacciones de competencia y el canibalismo dentro del gremio, al construir sus telas en diferentes estratos y obtener de ese modo distintos recursos alimenticios (Harwood \& Obrycki, 2005). Este aspecto contribuye a la tendencia del uso de las arañas en el control biológico de plagas, ya que al utilizar diferentes estratos para la fijación de telas, la predación de insectos perjudiciales es más fiable.

Por su parte, las arañas Lycosidae presentan patrones específicos de distribución temporo-espacial que son considerados como estrategias para evitar la competencia interespecífica (Foelix 1996). Rubio et al. (2007) aseveran que $L$. humilis tiene una distribución espacial segregada lo que podría evitar la competencia intraespecifica. En este estudio se observó varias especies de licósidos durante todos los meses del muestreo, con picos de abundancia en diferentes meses.

Mediante este trabajo ofrecemos un análisis más profundo de las comunidades de arañas en estos cultivos, lo cual estimamos que será un valioso aporte para la implementación de dicho taxón en el control biológico de plagas.

Pese a que la conformación física del ambiente limita la composición de las comunidades de arañas, al influir en los sitios de refugio, reproducción y alimentación (AchitteSchmutzler et al., 2016), las modificaciones del ambiente como los cultivos de Citrus spp, tienden a albergar varias poblaciones de arañas que se adaptaron a dicho cambio. Finalmente, consideramos que la distribución de las arañas en el follaje, el tronco y el suelo del cultivo de C. sinensis estudiado, está consolidado espacial y temporalmente.

\section{RESUMEN}

Los estudios de la araneofauna en los agroecosistemas son cruciales para la implementación de las arañas como agentes potenciales de control biológico de plagas. En este trabajo se analiza la distribución del ensamble de arañas en los microhábitats (follaje, tronco y suelo) de los cultivos de Citrus sinensis, (Rutaceae) de la Provincia de Corrientes, Argentina. Los muestreos se realizaron mensualmente mediante cuatro técnicas de captura: agitación de follaje, tamizado de hojarasca, pitfall y captura manual. En total se obtuvieron 2160 muestras y 7194 ejemplares de arañas (2462 en follaje, 983 en tallo y 3749 en suelo) pertenecientes a 35 familias y 200 especies de Araneomorphae. Hubo diferencias significativas en la estructura de las comunidades de arañas de los tres microhábitats y entre los meses, según el análisis de similitud (ANOSIM). 
El follaje presentó mayor diversidad, seguido por el suelo y el tronco. Araneidae, Theridiidae y Salticidae predominaron en el follaje; las especies dominantes fueron: Jessica erythostoma y Cheiracantium inclusum, esta araña es una de las principales depredadoras de las larvas de Phyllocnistis citrella (Lepidoptera) que atacan las hojas de Citrus. En el tronco abundaron: Tetragnathidae, Hersiliidae y Theridiidae, destacándose Iviraiva pachyura, una especie especialista en dicho microhabitat; y Leucauge venusta la cual consideramos que es una especie oportunista, debido a que su abundancia se incrementa notablemente en sitios modificados por el hombre. En el suelo abundaron: Lycosidae, Linyphiidae y Coriniidae; Lycosa erythrognatha fue la especie dominante, seguida de Pardosa plumipedata. El índice de similitud Chao-Jaccard fue mayor entre el follaje y el tronco $(\mathrm{J}-\mathrm{C}=0.95)$, asimismo el ordenamiento Escalado Multidimencional no Métrico (NMDS) mostró una clara diferenciación de la composición de especies del suelo con respecto al follaje y al tronco. Además, en el suelo hubo mayor recambio temporal de especies y el método de seriación resultó significativo $(\mathrm{z}=3.82, \mathrm{P}=0.00)$ por lo tanto hubo una sucesión de especies durante el año de muestreo. Las especies en cada microhabitat probablemente evitan la competencia al utilizar distintas estrategias de caza y recursos ecológicos, además de patrones específicos de distribución temporal. Nuestros resultados sugieren que las modificaciones del ambiente, como en estos cultivos de C. sinensis, tienden a albergar varias poblaciones de arañas que se adaptaron a dicho cambio e interactúan de manera equilibrada en espacio y tiempo.

Palabras clave: microhábitat; Araneae; agroecosistemas; naranjales; control biológico.

\section{REFERENCIAS}

Achitte-Schmutzler, H. C., Avalos, G., \& Oscherov, E. B. (2016). Comunidades de arañas en dos localidades del sitio RAMSAR Humedales Chaco, Argentina. Cuadernos de Investigación UNED, 8(2), 115-121.

Almada, M. S. (2014). Biodiversidad y densidad de arañas (Araneae) en un sistema agropastoril, tendientes a mejorar el impacto de los enemigos naturales sobre insectos plaga (Tesis doctoral). Facultad de Ciencias Naturales y Museo, La Plata, Argentina.

Almada, M. S., \& Sarquis, J. A. (2016). Araneofauna (Arachnida: Araneae) de suelo en bosques nativos, exóticos y pajonales del Parque General San Martín, Entre Ríos Argentina. Ecología Austral, 26, 286-292.

Almada, M. S., Sosa, M. A., \& González, A. (2012). Araneofauna (Arachnida: Araneae) en cultivos de algodón (Gossypium hirsutum) transgénicos y convencionales en el norte de Santa Fe, Argentina. Revista de Biología Tropical, 60(2), 611-623.
Amalin, D. M., \& Peña, J. E. (1999). Predatory spiders in lime orchards and their importance in the control of citrus leafminer, Phyllocnistis citrella (Lepidoptera: Gracillariidae). Proceedings of the Florida State Horticultural Society, 112, 222-224.

Amalin, D. M., Peña, J. E., \& McSorley, R. (1996). Abundance of spiders in lime groves and their potential role in suppressing the citrus leafminer population. In M. A., Hoy (Ed.), Proceedings, International Meeting: Managing the Citrus Leafminer (pp. 23-25). Orlando, Florida: University of Florida.

Amalin, D. M., Peña, J. E., McSorley, R., Browning, H. W., \& Crane, J. H. (2001). Comparison of different sampling methods and effect of pesticide application on spider populations in line orchard in South Florida. Environmental Entomology, 30, 1021-1027.

Argov, Y., \& Rossler, Y. (1996). Introduction, Release and Recovery of Several Exotic Natural Enemies for Biological Control of the Citrus Leafminer, Phyllocnistis citrella, in Israel. Phytoparasitica, 24, 33-38.

Armendano, A., \& González, A. (2011). Efecto de las arañas (Arachnida: Araneae) como depredadoras de insectos plaga en cultivos de alfalfa (Medicago sativa) (Fabaceae) en Argentina. Revista de Biología Tropical, 59(4), 1651-1662.

Avalos, G., Achitte-Schmutzler, H. C., \& De los Santos, M. E. (2018). Caracterización de la fauna de arañas en monocultivos de Eucalyptus y Pinus de la Reserva del Iberá, Corrientes, Argentina. Revista Mexicana de Biodiversidad, 89, 134-148.

Avalos, G., Bar, M. E., Oscherov, E. B., \& González, A. (2013). Diversidad de Araneae en cultivos de Citrus sinensis (Rutaceae) de la Provincia de Corrientes, Argentina. Revista de Biología Tropical, 61(3), 1243-1260.

Avalos, G., Damborsky, M. P., Bar, M. E., Oscherov, E. B., \& Porcel, E. A. (2009). Composición de la fauna de Araneae (Arachnida) de la Reserva Provincial Iberá, Corrientes, Argentina. Revista de Biología Tropical, 57(1-2), 339-351.

Avalos, G., Oscherov, E. B., \& González, A. (2015). Preferencias alimentarias de Araneus uniformis (Araneae: Araneidae) y Jessica erythrostoma (Araneae: Anyphaenidae) en condiciones de laboratorio. Revista Ibérica de Aracnología, 27, 109-109.

Bale, J. S., van Lenteren, J. C., \& Bigler, F. (2008). Biological control and sustainable food production. Philosophical Transactions of the Royal Society B, 363, 761-776.

Barrientos, J. A., Villalba, M., Alvis-Davila, L., \& GarciaMar, F. (2010). Identification and abundance of spiders (Araneae) in citrus crops of Valencia. Boletín de Sanidad Vegetal. Plagas, 36, 69-85. 
Begon, M., Harper, J. L., \& Townsend, C. R. (1995) Ecología: Individuos, poblaciones y comunidades. Barcelona: Ediciones Omega.

Benamú, M. A. (2004). Estudio Comparativo de la diversidad de arañas de un campo en abandono y un cultivo convencional de limonero (Citrus limon L. Burm) en Rincón del Cerro, Montevideo, Uruguay (Tesis de Maestría). Universidad de la República, Montevideo, Uruguay.

Breene, G. R., Dean, D. A., \& Meagher, R. L. (1993). Spiders and ants of Texas citrus groves. Florida Entomology, 76, 168-170.

Brower, J. C., \& Kyle, K. M. (1988). Seriation of an original data matrix as applied to palaeoecology. Lethaia, 21, 79-93.

Cabrera, A. L., \& Willink, A. (1973). Biogeografía de América Latina. Monografía 13, Serie de Biología. Washington, D. C., EE.UU: Organización de Estados Americanos (OEA).

Cardoso, P., Pekár, S., Jocqué, R., \& Coddington, J. A. (2011). Global patterns of guild composition and functional diversity of spiders. Plos One 6(6): e21710. DOI: 10.1371/journal.pone.0021710

Carroll, D. P. (1980). Biological notes on the spiders of some citrus groves in central and southern California. Entomological News, 91(5), 147-154.

Chao, A., Chazdon, R. L., Colwell, R. K., \& Shen, T. J. (2005). A new statistical approach for assessing similarity of species composition with incidence and abundance data. Ecology Letters, 8, 148-159.

Clarke, K. R. (1993). Non-parametric multivariate analysis of changes in community structure. Australian Journal of Ecology, 18, 117-143.

Colwell, R. K. (2013). EstimateS: statistical estimation of species richness and shared species from samples (Versión 9.1.0). Recuperado de http://viceroy.eeb. uconn.edu/estimates/

Colwell, R. K., Mao, C. X., \& Chang, J. (2004). Interpolating, extrapolating, and comparing incidencebased species accumulation curves. Ecology, 85, 2717-2727.

Costa, F. G., Pérez-Miles, F., Gudynas, E., Prandi, L., \& Capocasale, R. (1991). Ecología de los arácnidos criptozoicos, excepto ácaros, de Sierra de las Ánimas (Uruguay). Ordenes y Familias. Aracnología, $13 / 15: 1-41$

Danza, A. (2017). Perfil de mercado de cítricos dulces. Subsecretaria de mercados agropecuarios. Argentina: Ministerio de Agroindustria, Presidencia de la Nación. Recuperado de https://www.agroindustria.gob.ar/ sitio/areas/ss_mercados_agropecuarios/areas/frutas/ archivos/000030 Informes/100007 Perfil\%20 de $\% 20$ Mercado/000008 Perfil\%20del\%20mercado $\% 20 \mathrm{de} \% 20 \mathrm{c} \% \mathrm{C3} \%$ ADtricos\%20dulces\%202017. pdf

Dean, D. A., Sterling, W. L., \&. Horner, N. V. (1982). Spiders in eastern Texas cotton fields. Journal of Arachnology, 10, 251-260.

Díaz Porres, M., Rionda, M. H., Duhour, A. E., \& Momo, F. R. (2014). Artrópodos del suelo: Relaciones entre la composición faunística y la intensificación agropecuaria. Ecología Austral, 24, 327-334.

Eisner, T., Alsop, R., \& Ettershank, P. (1964). Adhesiveness of spider silk. Science, 146, 1058-1061.

Elizondo-Solís, J. M. (2002). Inventario y fluctuación poblacional de insectos y arañas asociadas con Citrus sinensis en la región Huetar Norte de Costa Rica. Manejo integrado de plagas y Agroecología, 64, 88-98.

Escobar, M. J., Avalos, G., \& Damborsky, M. P. (2012). Diversidad de Araneae (Arachnida) en la Reserva Colonia Benítez, Chaco Oriental Húmedo, Argentina. Revista FACENA, 28, 3-17.

Florez, D. E. (2000). Comunidad de arañas de la región Pacífica del departamento del valle del Cauca, Colombia. Revista Colombiana de Entomología, 26, $77-81$.

Foelix, R. F. (1996). Biology of Spiders. New York, Estados Unidos: Oxford University Press.

Garijo, C., \& García, E. J. (1994). Phyllocnistis citrella (Stainton, 1856) (Insecta: Lepidoptera: Gracillariidae: Phyllocnistinae) en los cultivos cítricos de Andalucía (sur de España): Biología, ecología y control de la plaga. Boletín de Sanidad Vegetal Plagas, 20, 815-826.

Gómez, C. A. (2016). Malezas en plantaciones cítricas: características y métodos de control. Instituto Nacional de Tecnología Agropecuaria, INTA. Recuperado de https://inta.gob.ar/documentos/malezas-en-plantaciones-citricas-caracteristicas-y-metodos-de-control

Grismado, C. J., Crudele, I., Damer, L., López, N., Olejnik, N., \& Trivero, S. (2011). Comunidades de arañas de la Reserva Natural Otamendi, Provincia de Buenos Aires. Composición taxonómica y riqueza específica. Revista Biológica, 14, 7-48.

Hammer, O., Harper, D. A. T., \& Ryan, P. D. (2001). PAST: palaeontological statistics (Versión 2.17). Recuperado de http:// folk.uio.no/ohammer/past

Harwood, J. D., \& Obrycki, J. J. (2005). Quantifying aphid predation rates of generalist predators in the field. European Journal of Entomology, 102, 335-350.

Henschell, J. R., \& Lubin, Y. D. (1997). A test of habitat selection at two spatial scales in a sit and wait 
predator: A web spider in the Naimb Desert Dunes. Journal Animal Ecology, 66, 401-413.

Heublein, D. (1983). Räumliche Verteiung, Biotoppräferenzen und kleinräumige Wanderungen der epigäischen Spinnen-fauna eines Wald-Wiesen-Ökotons; ein Breitag zum Thema "Randeffekt". Zoologische Jahrbücher (Systematik), 110, 473-519.

Jackson, D. A., Peres-Neto, P. R., \& Olden, J. D. (2001). What controls who is where in freshwater fish communities - the roles of biotic, abiotic, and spatial factors. Canadian Journal of Fisheries and Aquatic Sciences, 58, 157-170.

Jiménez, M. L., \& Tejas, A. (1996). Variación temporal de la araneofauna en frutales de la región del Cabo, Baja California Sur. México. Southwestern Entomolology, 21, 331-335.

Jiménez-Valverde, A., \& Hortal, J. (2003). La curva de acumulación de especies y la necesidad de evaluar los inventarios biológicos. Revista Ibérica de Aracnología, 8, 151-161.

Kacar, G. (2015). Biodiversity of Spider Species, Interactions with Horticultural Crops and a New Record for Turkey. Pakistan Journal of Zoology, 47(2), 545-550.

Keswani, S. (2014). Diversity, population and microhabitat used by spiders in citrus agroecosystem. Indian Society of Arachnology, 3(2), 90-101.

Krell, F. T. (2004). Parataxonomy versus taxonomy in biodiversity studies-pitfalls and applicability of 'morphospecies' sorting. Biodiversity and Conservation, $13,795-812$.

Lee, J. H., \& Kim, S. T. (2001). Use of spiders as natural enemies to control rice pest in Korea. Retrieved from: http://ag.udel.edu/delpha/ 8323

Mansour, F., Ross, J., Edwards, G., Whitcomb, W. H., \& Richman, D. (1982). Spiders of Florida Citrus groves. Florida Entomology, 65, 514-522.

Mansour, F., \& Whitcomb, W. H. (1986). The spiders of a citrus grove in Israel and their role as biocontrol agents of Ceroplastes floridensis [Homoptera: Coccidae]. Entomophaga, 31, 269-276.

Marín Loayza, R., Herrera, J. A., \& Páez, J. N. (2013). Biodiversidad microtemporal de la aracnofauna en el agrosistema del olivo cultivar Arauco en La Rioja, Argentina. UNLaR Ciencia, 1(1), 15-18.

Martin, T. J., \& Major, R. E. (2001). Changes in wolf spider (Araneae) assemblages across woodland-pasture boundaries in the central wheat-belt of New South Wales, Australia. Austral Ecology, 26, 264-274.

McDaniel, S. G., \& Sterling, W. L. (1982). Predation of Heliothis virescens (F.) Eggs on Cotton in East Texas. Environmental Entomology, 11, 60-66.
Michel, A. K., \& Winter, S. (2009). Tree microhabitat structures as indicators of biodiversity in Douglas-fir forests of different stand ages and management histories in the Pacific Northwest, U.S.A. Forest Ecology and Management, 257, 1453-1464.

Molina, N., Lombardo, E., Ramírez, A., Vallejos, A., Volpato, L., \& Zubrzycki, H. (2005). Informe citrícola de la Provincia de Corrientes. INTA EEA Bella Vista, 29, 1-9.

Montti, M. I., Visciglio, S. B., Raviol, F. H., Subovich, G. E., \& Munitz, M. S. (2013). Incidencia de la carga inicial de pesticidas en fruta sobre los niveles residuales en aceites esenciales cítricos. Ciencia, Docencia y Tecnología, 24(47), 187-218.

Monzó, C., Mollá, O., Castañera, P., \& Urbaneja, A. (2009). Activity-density of Pardosa cribata in Spanish citrus orchards and its predatory capacity on Ceratitis capitata and Myzus persicae. BioControl, 54, 393-402.

Moreno, C. E. (2001). Manual de métodos para medir la biodiversidad. Xalapa, México: Universidad Veracruzana.

Morrone, J. J. (2001). Biogeografía de América Latina y el Caribe. Zaragoza, España: M\&T-Manuales \&Tesis SEA.

Nentwig, W. (1987). The prey of spiders. In W. Nentwig (Ed.), Ecophysiology of Spiders (pp. 249-263). Berlin, Heidelberg: Springer-Verlag.

Nentwig, W. (1993). Spiders of Panama. Fauna \& Flora Handbook $N^{o} 12$. Florida, USA: The Sandhill Crane Press.

Nyffeler, M., \& Benz, G. (1981). Field studies on the feeding ecology of spiders: observations in the region of Zurich (Switzerland). Anzeiger für Schäedlingskunde, Pflanzenschutz, Umweltschutz, 54, 33-39.

Nyffeler, M., \& Benz, G. (1989). Foraging ecology and predatory importance of a guild of orb-weaving spiders in a grassland habitat. Journal of Applied Entomology, 107, 166-184.

Nyffeler, M., Sterling, W., \& Dean, D. (1994). Insectivorus activities of spiders in United States field crops. Journal of Applied Entomology, 118, 113-128.

Orellana, M. O., Ávila, I. H., \& Estrada, P. M. (2012). Diversity of spiders in an almond Prunus dulcis (Mill.) D.A. Webb orchard in the Metropolitan Region of Chile (Central Chile). Idesia, 30(1), 17-24. DOI: $10.4067 / \mathrm{S} 0718-34292012000100003$

Patrick, M. \& Canard, A. (1997). Maintaining spider biodiversity in agroecosystems as a tool in pest control. Agriculture Ecosystems \& Environment, 62(2-3), 229-235. 
Pearce, S., \& Zalucki, M. P. (2006). Do predators aggregate in response to pest density in agroecosystems? Assessing within-field spatial patterns. Journal of Applied Ecology, 43, 128-140.

Pérez-Miles, F., Simó, M., Toscano-Gadea, C., \& Useta, G. (1999). La comunidad de Araneae criptozóicas del Cerro de Montevideo, Uruguay: Un ambiente rodeado por urbanización. Physis, 57, 73-78.

Quijano, L. C., \& Neis Martínez, H. (2015). Variación temporal de la araneofauna (Arachnida: Araneae) en un fragmento de bosque seco tropical (bst), en el Departamento del Atlántico, Colombia. Boletín Científico Museo de Historia Natural, 19(2), 381-396.

Ramírez, M. J. (1999). Orden Araneae. En F. A Crespo, M. S. Iglesias, \& A. C. Valverde (Eds.), El ABC en la determinación de artrópodos. Claves para especímenes presentes en la Argentina I (pp. 39-59). Buenos Aires, Argentina: Editorial CCC Educando.

Riechert, S. E., \& Lockley, T. (1984). Spiders as biological control agents. Annual Review of Entomology, 29, 299-320.

Rodrigues, E. N. L., Mendonca Jr., M. de S., Rodrigues, P. E. S., \& Ott, R. (2015). Diversity, composition and phenology of araneid orb-weavers (Araneae, Araneidae) associated with riparian forests in southern Brazil. Iheringia, Série Zoologia, 105(1), 53-61.

Rubio, G. D., Corronca, J. A., \& Damborsky, M. P. (2008). Do spider diversity and assemblage change on different contiguous habitats? A case on protected habitats of Humid Chaco Ecoregion, North-East of Argentina. Environmental Entomology, 37(2), 419-430.

Rubio, G. D., Minoli, I., \& Piacentini, L. (2007). Patrones de abundancia de cinco especies de arañas lobo (Araneae: Lycosidae) en dos ambientes del Parque Nacional Mburucuyá, Corrientes, Argentina. Brenesia, 67, 59-67.

Ruzicka, V. (1987). Biodiagnistic evaluation of epigeic spider communities. Ekológia (CSSR), 6(4), 345-357.

Schmidt, M. H., \& Tscharntke, T. (2005). Landscape context of sheetweb spider (Araneae: Linyphiidae) abundance in cereal fields. Journal of Biogeography, $32,467-473$.
Sokal, R. R., \& Rohlf, F. J. (1995). Biometry: The principles and practice of statistics in biological research. New York: W.H. Freeman and Company.

Sorensen, L. L., Coddington, J. A., \& Scharff, N. (2002). Inventorying and estimating subcanopy spider diversity using semiquantitative sampling methods in an Afromontane forest. Environmental Entomology, 31(2), 319-330.

Szinetar, C., \& Horvath, R. (2005). A review of spiders in tree trunks in Europe (Araneae). Acta Zoologica Bulgarica Supplementum, 1, 221-257.

Toti, D. S., Coyle, F. A., \& Miller, J. A. (2000). A structured inventory of Appalachian grass bald and heath bald spider assemblages and a test of species richness estimator performance. Journal of Arachnology, 28, 329-345.

Turnbull, A. L. (1960). The spider population of a stand of oak (Quercus robur L.) in Wytham Wood, Berks., England. Entomological Society of Canada, 92, 110-124.

Uetz, G. W. (1976). Gradient analysis of spider communities in a streamside forest. Oecologia, 22, 373-385.

Van Den Berg, A. M., Dippenaar-Schoeman, A. S., Deacon, V. E., \& Anderson, S. H. (1992). Interactions between Citrus Psylla, Trioza erytreae (Hem. Triozidae), and spiders in an unsprayed Citrus orchard in the Transvaal Lowveld. Entomophaga, 37, 599-608.

Wise, D. H. (1993). Spiders in Ecological Webs. Cambridge. Inglaterra: Cambridge University Press.

Wolda, H. (1988). Insect seasonality: why? Annual Review of Ecology and Systematics, 19, 1-18.

World Spider Catalog. (2018). World Spider Catalog (Version 19.0). Natural History Museum Bern. Recuperado de http://wsc.nmbe.ch

Ziesche, T. M., \& Roth, M. (2008). Influence of environmental parameters on small-scale distribution of soil-dwelling spiders in forests: What makes the difference, tree species or microhabitat? Forest Ecology and Management, 255, 738-752. 